\title{
Upright Integrated Relaxation Pressure Predicts Symptom Outcome for Esophagogastric Junction Outflow Obstruction
}

\author{
Songfeng Chen, ${ }^{1}$ Mengya Liang, ${ }^{2}$ Niandi Tan, ${ }^{1}$ Mengyu Zhang, ${ }^{1}$ Yuqing Lin, ${ }^{1}$ Peixian Cao, ${ }^{1}$ Qianjun Zhuang, ${ }^{1}$ and Yinglian Xiao $^{1 *}$ \\ Departments of ${ }^{1}$ Gastroenterology and ${ }^{2}$ Cardian Surgery, The First Affiliated Hospital, Sun Yat-sen University, Guangzhou, China
}

\section{Background/Aims}

Esophagogastric junction outflow obstruction (EGJOO) is characterized by elevated integrated relaxation pressure (IRP) and preserved esophageal peristalsis. The clinical significance of EGJOO is uncertain. This study aim to describe the clinical characteristics of these patients and to find out potential parameters to predict patients' symptom outcome.

\section{Methods}

Consecutive patients who received high-resolution manometry examination in our hospital in 2013-2019 and met the diagnostic criteria of EGJOO were retrospectively included. Motility and reflux parameters as well as endoscopy and barium esophagogram results were studied and compared. Patients were also followed up to record their treatment methods and symptom outcomes.

\section{Results}

A total of 138 EGJOO (accounting for $5.2 \%$ of total patients taking high-resolution manometry examination in our hospital) patients were included. Only $2.9 \%$ of these patients had persistent dysphagia. A total of $81.8 \%$ of EGJOO patients had symptom resolution during follow-up. Patients with persistent dysphagia had significantly higher upright $\operatorname{IRP}(16.6[10.3,19.8]$ vs $7.8[3.2,11.5] ; P=$ 0.026 ) than those without. Upright IRP can effectively distinguished patients with persistent dysphagia (area under curve: $0.826 ; P=$ 0.026) using optimal cut-off value of $9.05 \mathrm{mmHg}$.

\section{Conclusion}

EGJOO patients with persistent dysphagia and higher upright IRP (median $>9.05 \mathrm{mmHg}$ ) needs further evaluation and aggressive management.

(J Neurogastroenterol Motil 2021;27:363-369)

Key Words

Deglutition disorders; Esophagogastric junction; Manometry

\section{Introduction}

Esophagogastric junction outflow obstruction (EGJOO) is an entity of Chicago classification version 3.0 , which is characterized by elevated integrated relaxation ( $\geq 15 \mathrm{mmHg}$ ) in combination with preserved esophageal peristalsis. ${ }^{1}$ Although it was considered as a major motility disorder, little is known about its etiology and clinical

Received: May 12, 2020 Revised: None Accepted: December 19, 2020

(a) This is an Open Access article distributed under the terms of the Creative Commons Attribution Non-Commercial License (http://creativecommons. org/licenses/by-nc/4.0) which permits unrestricted non-commercial use, distribution, and reproduction in any medium, provided the original work is properly cited.

*Correspondence: Yinglian Xiao, MD, PhD

Department of Gastroenterology, The First Affiliated Hospital, Sun Yat-sen University, Guangzhou 510080, China Tel: +86-020-87755766 (ext. 8182), Fax: +86-020 87332916, E-mail: xyingl@mail.sysu.edu.cn 
significance. EGJOO is a heterogeneous motility diagnosis. The symptoms of patients with patients vary greatly. The most common chief complaints of these patients are heartburn, regurgitation, and dysphagia. ${ }^{2-5}$ In most cases, patients' symptoms can resolve spontaneously, ${ }^{4,6}$ with only a few progressing to achalasia. ${ }^{3}$ Its potential etiologies may include organic causes (eg, hiatus hernia, stricture, eosinophilic esophagitis, vascular obstruction, and malignancy) and functional causes (eg, early or incompletely express achalasia, esophageal wall stiffness, and drug effect). ${ }^{7}$ Most organic causes of EGJOO mentioned above can be identified by initial upper endoscopy. Barium esophagogram has long been utilized in the diagnosis of achalasia and the assessment of esophagogastric junction (EGJ) outflow. Some researchers even argued that EGJOO should be investigated further aggressively by endoscopic ultrasonography, functional luminal imaging probe, or $\mathrm{CT}^{4,8-10}$ Despite the variety of means to evaluate EGJOO, none of current methods are known to be able to predict patients' symptom outcome.

The treatment of EGJOO is controversial. The majority of patients with EGJOO were given either no specific intervention or Botox injection. Some patients were treated by medication and a few patients with persistent dysphagia by surgery. However, the treatment outcomes were similar in these approaches. ${ }^{6}$ Therefore, it is necessary to come up with measures to select appropriate patients with EGJOO for aggressive treatment.

This study hypothesized that EGJOO is a heterogeneous disease that need to be further evaluated by endoscopy, barium esophagogram, and other parameters of high-resolution manometry (HRM). The current study aim to describe the clinical characteristics of patients with EGJOO and to find out potential parameters to predict patients' symptom outcome.

\section{Materials and Methods}

\section{Study Subjects}

Consecutive patients who received HRM examination in our hospital from January 2013 to December 2019 and diagnosed with EGJOO were retrospectively included. The exclusion criteria were: (1) gastroesophageal tumor history (2) gastroesophageal surgery history. Their baseline characteristics and symptoms were recorded in a questionnaire in our center including body mass index, impaction dysphagia question score (IDQ), Eckert-score, and gastroesophageal reflux disease questionnaire (GERDQ). Phone calls were conducted to follow up these patients in terms of symptom outcomes. Meanwhile, patients' HRM, endoscopy, esophagogram, and multichannel impedance-pH monitoring (MII-pH) results were reviewed. This study was approved by the Ethical Review Board of Sun Yat-sen University (IRB No. 2019290), and was conducted following the concept of the Declaration of Helsinki.

\section{High-resolution Manometry}

HRM was performed after the upper endoscopy tests. The procedure was carried out as previously reported. ${ }^{11}$ Firstly, patients were required to stop swallowing for 30 seconds to record the basal pressure. Ten $5-\mathrm{mL}$ liquid swallows were then performed in the supine position as well as five $5-\mathrm{mL}$ liquid swallows in the upright position. Multiple rapid swallows (MRS) were administered at 1-2 second interval for 3 times both in the supine and upright position. ${ }^{12}$

HRM parameters analysis was conducted using Manoview analysis software (Medtronic Inc, Minneapolis, MN, USA). EGJOO was defined as a median lower esophageal sphincter (LES) integrated relaxation pressure (IRP) $\geq 15 \mathrm{mmHg}$ across 10 supine liquid swallows and could not meet the diagnosis criteria of achalasia. Median IRP, mean distal contractile integral (DCI), LES relaxation intrabolus pressure (IBP), maximum IBP, MRSDCI, MRS-IRP, MRS ratio of both supine and upright position were calculated. MRS ratio was defined as MRS-DCI/mean liquid swallow DCI. ${ }^{13}$ Abnormal upright IRP was defined as IRP in the upright position $>12 \mathrm{mmHg}$. $^{14}$

\section{Multichannel Impedance-pH Monitoring}

The reflux monitoring procedure was carried out as previously reported. ${ }^{11}$ Patients were asked to live at their normal routines, and to note down their symptoms, postures, and meals during monitoring.

Manual analysis was conducted by 2 certificated investigators using the Bioview Analysis software (Sandhill Scientific Inc, Highland Ranch, CO, USA). Meal periods were excluded while analyzing. Pathological distal reflux was defined as acid exposure time at 5 $\mathrm{cm}$ above $\mathrm{LES} \geq 4.2 \%$, or $\geq 6 \%$ in the definition of Lyon consensus, ${ }^{15}$ of the monitoring time. Other reflux parameters including: (1) reflux episode, (2) bolus clearance time, (3) bolus exposure percent time, and (4) symptom association probability were also collected.

\section{Symptom Outcome}

Patients were followed up until December 2019. Treatments of these patients were divided into (1) no specific treatment, (2) medication treatment (including antacid medication, neuromodulator, prokinetic agents, herbal medicine, or any other drugs for chief symptoms at standard dose for more than 8 weeks), and (3) specific 
Table 1. Baseline Characteristics of Esophagogastric Junction Outflow Obstruction Patients

\begin{tabular}{ll}
\hline Characteristics & Distribution \\
\hline Age $(\mathrm{yr})$ & $47.8(12.5)$ \\
Male & 32.6 \\
BMI $\left(\mathrm{kg} / \mathrm{m}^{2}\right)$ & $20.9(3.2)$ \\
IDQ score & $0.0(0.0,4.0)$ \\
GERDQ score & $8.0(6.0,9.0)$ \\
\hline
\end{tabular}

BMI, body mass index; IDQ score, impaction dysphagia question score; GERDQ score, gastroesophageal reflux disease questionnaire score.

Values are presented as mean (SD), \%, or median (interquartile range [IQR]).

surgery treatment. Patients whose chief symptoms relieved during the week before follow-up were considered as responders. Persistent dysphagia was defined as dysphagia that persists for more than 1 year or needs invasive treatment.

\section{Statistical Methods}

For categorical variables, the percentage form was adopted and chi-square tests were used for comparisons. The mean $\pm \mathrm{SD}$ of normally distributed continuous variables were presented, which were compared by $t$ test. Median (95\% confidence interval) and Mann-Whitney $U$ test were adopted for continuous data that were not normally distributed. Receiver operator characteristic curve was used to evaluate the efficacy of different parameters in predicting symptom outcomes and to determine the optimal cutoff of different indexes. The significance level ( $P$-value $)$ was set at 0.05 .

\section{Results}

A total of 138 patients (accounting for $5.2 \%$ of total patients taking HRM examination in our hospital, the demographic data was shown in Table 1) were diagnosed with EGJOO in 20132019. The chief complaints of patients with EGJOO were heartburn, regurgitation, globus, chest pain, and dysphagia (Fig. 1). Only $2.9 \%$ of patients with EGJOO had persistent dysphagia symptoms. Among all the patients with EGJOO, 80.4\% completed a reflux monitoring, those who did not undergo reflux monitoring were due to the lack of GERD symptoms and/or because they had severe dysphagia symptoms which made them unable to tolerate the ambulatory monitoring. Pathological reflux was found in $7.9 \%$ (or $5.9 \%$ as defined in Lyon consensus) of patients with EGJOO. As for endoscopy results, $12.2 \%$ of patients with EGJOO were diagnosed with esophagitis while only $0.7 \%$ had anatomical causes (vascular obstruction) for EGJOO. Only 36 patients completed the barium esophagogram, and 4 patients $(11.1 \%)$ were diagnosed with

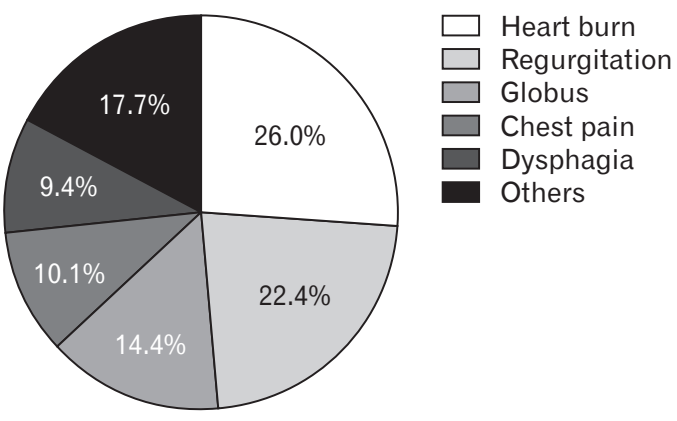

Figure 1. Chief complaints distribution of patients with esophagogastric junction outflow obstruction.

Table 2. Response Rates Comparison by Different Treatment Methods

\begin{tabular}{lcc}
\hline \multicolumn{1}{c}{ Treatment methods } & $\begin{array}{c}\text { Number of } \\
\text { patients (\%) }\end{array}$ & $\begin{array}{c}\text { Response } \\
\text { rate (\%) }\end{array}$ \\
\hline No specific treatment & $81(58.6)$ & $70(85.1)$ \\
Neuroregulator & $26(18.8)$ & $20(76.9)$ \\
Proton pump inhibitor & $18(13.0)$ & $12(66.6)$ \\
Other medication (mainly herbal medicine) & $10(7.2)$ & $9(90.0)$ \\
Botox injection & $0(0.0)$ & - \\
Pneumatic dilation & $0(0.0)$ & - \\
Peroral endoscopic myotomy & $3(2.9)$ & $3(100.0)$ \\
\hline
\end{tabular}

achalasia (according to bird's-beak appearance).

Most patients had benign symptom outcomes. The overall response rate of patients with EGJOO was $81.8 \%$. More than half of patients with EGJOO did not receive any specific treatment but the symptoms relieved by itself. The most commonly used drugs were neuromodulator (eg, tricyclic antidepressants and serotonin norepinephrine reuptake inhibitors) and proton pump inhibitors. Response rates of different treatment methods were shown in Table 2.

\section{Comparison by Symptoms}

Patients were divided into dysphagia group $(\mathrm{n}=13)$ and nondysphagia group ( $n=125$ ) according to their chief complaints. Dysphagia group had significantly higher IDQ scores (Table 3). No significant difference was observed in gender, age, body mass index, and GERDQ scores (Table 3).

As for endoscopy, patients with chief complaint of dysphagia were more likely to have organic causes for EGJOO (Table 3). Seven patients in the dysphagia group (53.8\%) and 26 patients in the non-dysphagia (20.8\%) group completed a barium esophagogram. EGJOO patients with chief complaint of dysphagia were more likely to be diagnosed with achalasia by barium esophagogram (Table 3). 
Patients with dysphagia were found to have higher IRP both in supine (20.7 [17.8, 22.2] vs 17.5 [16.3, 19.3]; $P=0.008$; Fig. 2A) and upright positions $(10.4[8.6,13.7]$ vs. $7.4[3.1,11.6] ; P=0.012$; Fig. 2B). No significant differences were found in other motility parameters. No significant differences were found in reflux parameters between the dysphagia group and the non-dysphagia group either.

Considering that some patients had dysphagia symptoms relieved by itself, persistent dysphagia symptom may be more clinically significant. Therefore, the samples were further divided into 2 groups: (1) patients with persistent dysphagia (dysphagia that persists for more than 1 year or needs invasive treatment) and (2)

Table 3. Baseline Characteristics Comparison by Chief Complaints

\begin{tabular}{|c|c|c|c|}
\hline Characteristics & Dysphagia group $(\mathrm{n}=13)$ & Non-dysphagia group $(\mathrm{n}=125)$ & $P$-value \\
\hline Age (yr) & $45.9(13.8)$ & $48.0(12.4)$ & 0.560 \\
\hline Male & 23.1 & 33.6 & 0.646 \\
\hline $\operatorname{BMI}\left(\mathrm{kg} / \mathrm{m}^{2}\right)$ & $19.8(3.4)$ & $21.0(3.2)$ & 0.218 \\
\hline IDQ score & $8.0(1.0,10.5)$ & $0.0(0.0,3.0)$ & $<0.001$ \\
\hline GERDQ score & $6.0(6.0,9.5)$ & $8.0(6.0,9.0)$ & 0.631 \\
\hline \multicolumn{4}{|l|}{ Endoscopy } \\
\hline Esophagitis & 0.0 & 13.6 & 0.328 \\
\hline Vascular obstruction & 7.6 & 0.0 & 0.029 \\
\hline \multicolumn{4}{|l|}{ Esophagogram } \\
\hline Achalasia (diagnosed by esophagogram) & 57.1 & 0.0 & $<0.001$ \\
\hline
\end{tabular}

BMI, body mass index; IDQ score, impaction dysphagia question score; GERDQ score, gastroesophageal reflux disease questionnaire score.

Values are presented as mean $(\mathrm{SD}), \%$, or median (interquartile range $[\mathrm{IQR}]$ ).
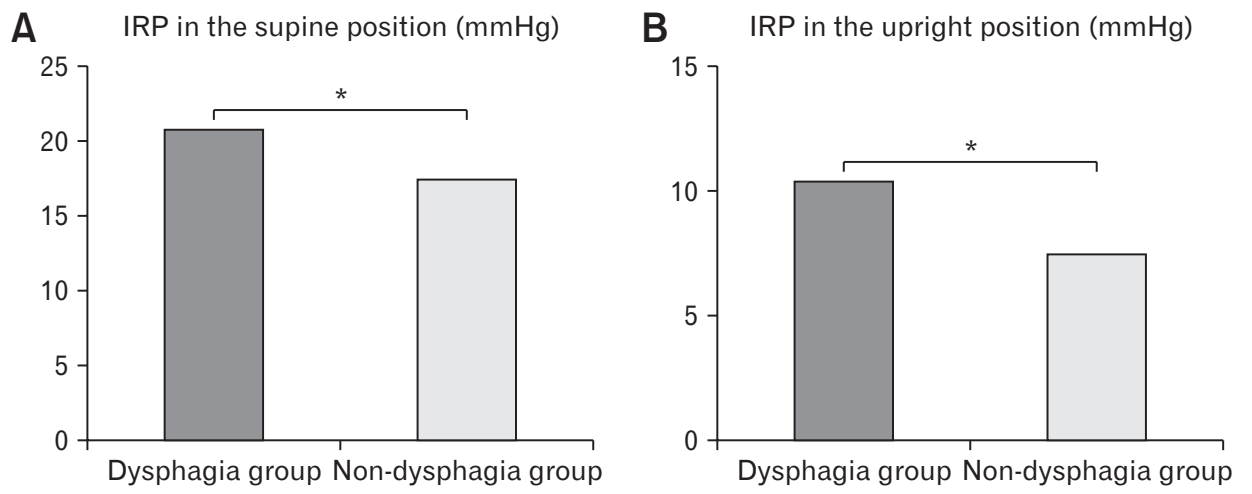

Figure 2. Motility parameters comparison by chief symptoms. IRP, integrated relaxation pressure. ${ }^{*} P<0.05$.

A

IRP in the upright position $(\mathrm{mmHg})$

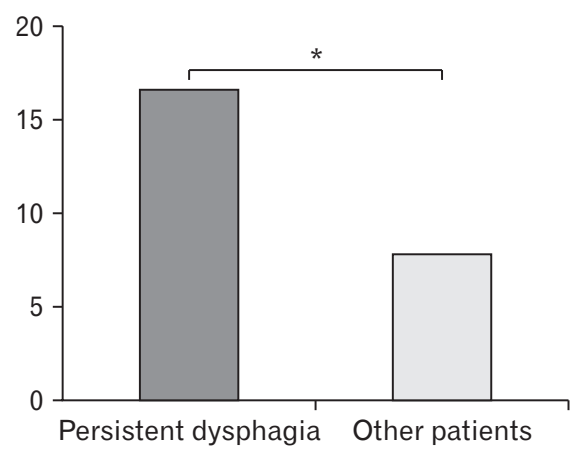

B

Ratio of abnormal IRP in the upright position (\%)

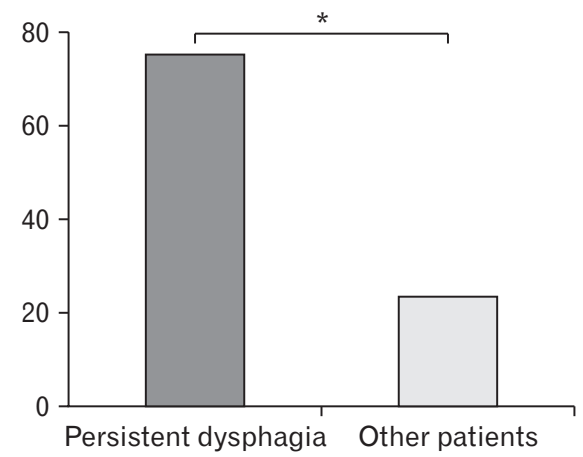

Figure 3. Motility parameters comparison by patients with and without persistent dysphagia. IRP, integrated relaxation pressure. ${ }^{*} P<0.05$. 
patients without persistent dysphagia. Patients with persistent dysphagia ( $\mathrm{n}=4$; 3 of them received peroral endoscopic myotomy treatment while the other 1 refused to receive any specific treatment) were more likely to have organic causes for EGJOO than other patients $(25.0 \%$ vs $0.0 \% ; P<0.001)$. They were also more likely to be diagnosed with achalasia by barium esophagogram (100.0\% vs $0.0 \% ; P<0.001)$. EGJOO patients with persistent dysphagia were found to have significantly higher upright IRP (16.6 $[10.3,19.8]$ vs $7.8[3.2,11.5] ; P=0.026$; Fig. $3 \mathrm{~A})$ and abnormal upright IRP ratio ( $75.0 \%$ vs $18.8 \%$; $P=0.031$; Fig. 3B). Receiver operator characteristic curve was used to evaluate the efficacy of different HRM parameters in predicting persistent dysphagia. Upright IRP effectively distinguished the prognosis of patients (area under curve: $0.826 ; P=0.026$ ), with the optimal cut-off of $9.05 \mathrm{mmHg}$ (Fig. 4).

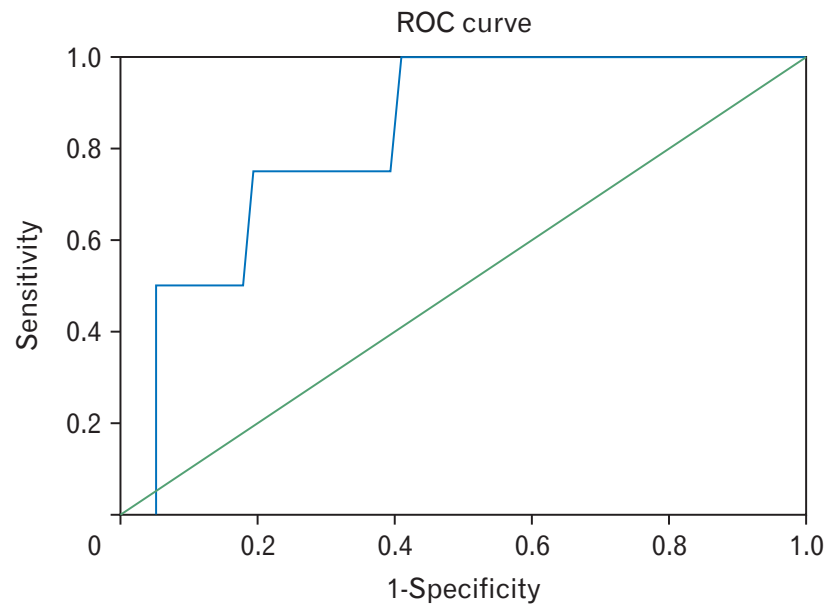

Figure 4. Receiver operator characteristic (ROC) curve of upright integrated relaxation pressures (IRP) in predicting persistent dysphagia.

\section{Comparison by Barium Esophagogram}

Thirty-six patients in total had completed a barium esophagogram. Four patients (11.1\%) were diagnosed with achalasia (3 of them received peroral endoscopic myotomy treatment while the other 1 refused to receive any specific treatment), while the other 32 patients with EGJOO were considered normal. Patients were divided into the achalasia group and the non-achalasia group based on their barium esophagogram results. Patients in the achalasia group were found to have significantly higher IDQ scores, and were more likely to have dysphagia or persistent dysphagia symptoms (Table 4). Compared with patients in the non-achalasia group, more patients in the achalasia group were found to have organic causes for EGJOO during endoscopy examination (Table 4). As for HRM, patients in the achalasia group had significantly higher upright IRP $(16.6[10.3,19.8]$ vs $7.9[4.3,12.8] ; P=$ 0.012). Since none of these achalasia patients underwent MII-pH, comparison of reflux parameters was not available.

\section{Discussion}

With the widespread application of HRM, EGJOO is no longer a rare motility diagnosis in clinic. Former studies suggested that EGJOO accounts for $8.2-24.3 \%$ of total patients taking HRM examination. ${ }^{3,4,14,16}$ Although it was considered as a major motility disorder, most patients diagnosed with EGJOO were dysphagiafree, not to speak of persistent dysphagia. ${ }^{2-5}$ Therefore, whether EGJOO is clinically significant remains controversial. The current study was designed to describe the clinical characteristics of these patients. In this study, only $9.4 \%$ of patients with EGJOO had a chief complaint of dysphagia, and $11.6 \%$ of patients had some

Table 4. Baseline Characteristics Comparison by Barium Esophagogram Diagnoses

\begin{tabular}{lccr}
\hline \multicolumn{1}{c}{ Characteristics } & Achalasia group $(\mathrm{n}=4)$ & Non-achalasia group $(\mathrm{n}=32)$ & $P$-value \\
\hline Age $(\mathrm{yr})$ & $42.8(24.7)$ & $48.2(11.5)$ & 0.446 \\
Male & 75.0 & 31.2 & 0.244 \\
BMI $\left(\mathrm{kg} / \mathrm{m}^{2}\right)$ & $20.9(3.6)$ & $20.8(2.1)$ & 0.974 \\
IDQ score & $7.0(10.0,13.8)$ & $0.0(0.0,4.8)$ & 0.005 \\
GERDQ score & $6.0(6.0,8.3)$ & $7.5(6.0,9.0)$ & 0.327 \\
Dysphagia & 100.0 & 9.3 & $<0.001$ \\
Persistent dysphagia & 100.0 & 0.0 & $<0.001$ \\
Endoscopy & & & 0.390 \\
Esophagitis & 0.0 & 9.3 & 0.031 \\
Vascular obstruction & 25.0 & 0.0 & \\
\hline
\end{tabular}

BMI, body mass index; IDQ score, impaction dysphagia question score; GERDQ score, gastroesophageal reflux disease questionnaire score. Values are presented as mean $(\mathrm{SD}), \%$, or median (interquartile range [IQR]). 
extent of dysphagia. Most patients with EGJOO had normal endoscopy, barium esophagogram results and most, whether having received specific treatment or not, had benign symptom outcomes. With regards to parameters that is possibly pertinent to symptom outcomes, the current study found that persistent dysphagia symptoms were relevant to elevated upright IRP as well as to abnormal EGJ findings in endoscopy and barium esophagogram.

Former studies have found that most patients with EGJOO had benign clinical outcomes. ${ }^{2,10,17,18}$ A retrospective study by PérezFernández et $\mathrm{al}^{10}$ reviewed 44 patients with EGJOO and found that over one-third of patients presented a spontaneous resolution of symptoms without specific treatment. Another study retrospectively included 83 patients with EGJOO and found that only $2.7 \%$ patients with EGJOO evolved to achalasia (median follow-up time: 6 months). ${ }^{2}$ EGJOO diagnosis is based on the supine swallows. However, physiologically, drinking and eating are usually done in the upright position. In the recumbent position, EGJ may be compressed by other organs, which may elevate the EGJ pressure. Nevertheless, compression may be relieved in the upright position. This may partly explained the benign symptom outcomes and normal upright IRP of most patients with EGJOO.

Despite the benign outcome of EGJOO, it is important to screen out patients who need aggressive management. A study published by Ong et $\mathrm{al}^{4}$ retrospectively included 40 patients with EGJOO and found that $73.5 \%$ of functional patients with EGJOO had spontaneous resolution of their symptoms and dysphagia may be a signal of further evaluation. Poong-Lyul Rhee analysed 169 patients with EGJOO detected by combined multichannel intraluminal impedance and HRM and found that suspected clinical significant patients are more likely to have dysphagia, compartmentalized pressurization, and abnormal liquid bolus transit. ${ }^{19}$ Another retrospective study found that Nadir upper esophageal sphincterresidual pressure may predict EGJOO patients' symptom outcome for myotomy. ${ }^{5}$ It should be noted that none of the studies mentioned above compared upright HRM parameters as well as reflux parameters. A recent study published by Triggs et $\mathrm{al}^{14}$ included 310 patients with EGJOO. Each patient performed 10 supine and 5 upright $5-\mathrm{mL}$ liquid swallows. It was found that the median upright IRP for patients with radiographic evidence of EGJOO or symptomatic dysphagia was higher than for patients without. In the current study, results of endoscopy, barium esophagogram, and reflux monitoring were all reviewed and compared. We also compared upright HRM parameters and some novel metrics such as MRS and IBP. In this study, symptoms of dysphagia especially persistent dysphagia were found to be correlated to elevated upright
IRP and abnormal EGJ outflow findings during esophagogram and endoscopy.

There were some limitations in our study. Firstly, only a few number $(26.1 \%)$ of patients with EGJOO conducted barium esophagogram. Secondly, few patients with EGJOO in this study had a repeated HRM test, leaving the question of whether elevated IRP can resolve spontaneously unconfirmed. Thirdly, only a small part patients in this study had dysphagia symptoms. Therefore, some parameters that help predict patients' symptom outcome may be missed due to the disproportion of sample size among groups. Last but not least, some of the patients with EGJOO included in this study were diagnosed with achalasia by esophagogram. The purpose of this study was to explore the clinical significance of patients with EGJOO diagnosed by HRM. Therefore, we did not exclude these patients in the current study. We conducted comparison by the results of the esophagograms. Patients with dysphagia symptoms and had higher upright IRP were more likely to be potential achalasia patients.

In conclusion, further evaluation (ie, esophagograms) and aggressive management should be considered in patients with EGJOO when patients report dysphagia symptoms and had significantly increased upright IRP (median $\geq 9.05 \mathrm{mmHg}$ ).

Financial support: The study was supported by grants from the National Natural Science Foundation of China (Grant No. 81770544 and 81970479) and the Medical Scientific Research Foundation of Guangdong Province of China (Grant No. A2019510).

\section{Conflicts of interest: None.}

Author contributions: Songfeng Chen: acquisition of data, analysis and interpretation of data, and drafting of the manuscript; Mengya Liang and Niandi Tan: interpretation of data drafting of the manuscript; Mengyu Zhang: analysis the data; Yuqing Lin, Peixian Cao, and Qianjun Zhuang: acquisition and interpretation of data; and Yinglian Xiao: study concept and design, analysis of data, finalizing and approving the manuscript, and guaranty of the article.

\section{References}

1. Kahrilas PJ, Bredenoord AJ, Fox M, et al. The Chicago classification of esophageal motility disorders, v3.0. Neurogastroenterol Motil 2015;27:160-174.

2. Lynch KL, Yang YX, Metz DC, Falk GW. Clinical presentation and disease course of patients with esophagogastric junction outflow obstruc- 
tion. Dis Esophagus 2017;30:1-6.

3. Liu A, Woo M, Nasser Y, et al. Esophagogastric junction outflow obstruction on manometry: outcomes and lack of benefit from CT and EUS. Neurogastroenterol Motil 2019;31:e13712.

4. Ong AML, Namasivayam V, Wang YT. Evaluation of symptomatic esophagogastric junction outflow obstruction. J Gastroenterol Hepatol 2018;33:1745-1750.

5. Blais P, Bennett MC, Gyawali CP. Upper esophageal sphincter metrics on high-resolution manometry differentiate etiologies of esophagogastric junction outflow obstruction. Neurogastroenterol Motil 2019;31:e13558.

6. Garbarino S, von Isenburg M, Fisher DA, Leiman DA. Management of functional esophagogastric junction outflow obstruction: a systemic review. J Clin Gastroenterol 2020;54:35-42.

7. Samo S, Qayed E. Esophagogastric junction outflow obstruction: where are we now in diagnosis and management? World J Gastroenterol 2019;25:411-417.

8. Kahrilas PJ, Bredenoord AJ, Fox M, et al. Expert consensus document: advances in the management of oesophageal motility disorders in the era of high-resolution manometry: a focus on achalasia syndromes. Nat Rev Gastroenterol Hepatol 2017;14:677-688.

9. Roman S, Kahrilas PJ. Challenges in the swallowing mechanism: nonobstructive dysphagia in the era of high-resolution manometry and impedance. Gastroenterol Clin North Am 2011;40:823-835, ix-x.

10. Pérez-Fernández MT, Santander C, Marinero A, Burgos-Santamaría D, Chavarría-Herbozo C. Characterization and follow-up of esophagogastric junction outflow obstruction detected by high resolution manometry. Neurogastroenterol Motil 2016;28:116-126.

11. Zhang M, Chen M, Peng S, Xiao Y. The Rome IV versus Rome III criteria for heartburn diagnosis: a comparative study. United European Gastroenterol J 2018;6:358-366.

12. Trudgill NJ, Sifrim D, Sweis R, et al. British society of gastroenterology guidelines for oesophageal manometry and oesophageal reflux monitoring. Gut 2019;68:1731-1750.

13. Shaker A, Stoikes N, Drapekin J, Kushnir V, Brunt LM, Gyawali CP. Multiple rapid swallow responses during esophageal high-resolution manometry reflect esophageal body peristaltic reserve. Am J Gastroenterol 2013;108:1706-1712.

14. Triggs JR, Carlson DA, Beveridge C, et al. Upright integrated relaxation pressure facilitates caracterization of esophagogastric junction outflow obstruction. Clin Gastroenterol Hepatol 2019;17:2218-2226, e2.

15. Gyawali CP, Kahrilas PJ, Savarino E, et al. Modern diagnosis of GERD: the lyon consensus. Gut 2018;67:1351-1362.

16. Triadafilopoulos G, Clarke JO. Clinical and manometric characteristics of patients with oesophagogastric outflow obstruction: towards a new classification. BMJ Open Gastroenterol 2018;5:e000210.

17. Okeke FC, Raja S, Lynch KL, et al. What is the clinical significance of esophagogastric junction outflow obstruction? evaluation of 60 patients at a tertiary referral center. Neurogastroenterol Motil 2017;29:e13061.

18. Schupack D, Katzka DA, Geno DM, Ravi K. The clinical significance of esophagogastric junction outflow obstruction and hypercontractile esophagus in high resolution esophageal manometry. Neurogastroenterol Motil 2017;29:1-9.

19. Song BG, Min YW, Lee H, et al. Combined multichannel intraluminal impedance and high-resolution manometry improves detection of clinically relevant esophagogastric junction outflow obstruction. J Neurogastroenterol Motil 2019;25:75-81. 